\title{
Four-year outcomes of unprotected left main lesion treated with one-stent versus two-stent technique
}

\author{
Lucian Predescu',2, Marin Postu', Lucian Zarma', Adrian Bucsa', Pavel Platon',2, Marian Croitoru', \\ Adrian Mereuta ${ }^{1,2}$, Leonard Lichiardopol' ${ }^{3}$, Alexandra Predescu4, Dan Mihai Dorobantu',5, \\ Carmen Ginghina',2, Dan Deleanu'
}

See page 363 for the editorial comment on this article.

\begin{abstract}
Introduction - Most reports on left main bifurcation lesions have demonstrated that treatment with a singlestent strategy is superior to a two-stent strategy but have excluded patients with acute coronary syndromes (ACS). Aims - The aim of the current study was to compare the four-year outcomes of patients with unprotected left main coronary artery disease (ULMCAD) treated by percutaneous coronary intervention $(\mathrm{PCl})$ with a one-stent or two-stent strategies in a population including those presenting as ACS. Methods - A total of I35 patients with ULMCAD treated by PCI were included, of which 75 (55.6\%) had a one-stent strategy (Group A) and 60 (44.4\%) had a two-stent strategy (Group B). Results - Fewer patients in Group A had a TIMI III flow at the end of the procedure $(89.4 \%$ vs $100 \%, p=0.03$ ) and complete revascularization $(65.3 \%$ vs $88.3 \%, p=0.002)$. We found a higher early mortality in Group A without reaching statistical significance $(13.4 \%$ vs $3.3 \%, p=0.1)$. Mortality rate at 4 -year follow up was higher with Group $A$ after multivariable analysis (adjusted HR 0.36, $\mathrm{Cl} 0.15-0.85, \mathrm{p}=0.02$ ). We found no significant differences between the groups in terms of major adverse cardiac event (MACE) (adjusted HR 0.85, Cl 0.34- I.48, $\mathrm{p}=0.7$ ) or target lesion revascularization (TLR) (adjusted $\mathrm{HR} \mathrm{I.37,} \mathrm{Cl}$ 0.42-4.47, $\mathrm{p}=0.6$ ) at 4-year follow up. Conclusions - Among unselected patients with ULMCAD PCl, with or without ACS, the early mortality rate is similar between one and two-stent strategy. Although, 4-year TLR and MACE rates were similar between the two groups, the 4-year all-cause mortality rate was lower in the two-stent strategy group.
\end{abstract}

Keywords: percutaneous coronary intervention, left main coronary artery disease, one-stent strategy, two-stent strategy.

Rezumat: Introducere - Cele mai multe rapoarte privind leziunile principale de bifurcaţie stângă au demonstrat că tratamentul cu o strategie cu un singur stent este superior unei strategii cu două stenturi, dar au exclus pacienții cu sindroame coronariene acute (ACS). Scop - Scopul studiului actual a fost compararea rezultatelor de patru ani ale pacienților cu boală coronariană principală stângă neprotejată (ULMCAD) tratată prin intervenție coronariană percutanată $(\mathrm{PCl})$ cu strategii cu un stent sau cu două stenturi într-o populație, inclusiv cele care prezintă ca ACS. Metode - Au fost incluși un număr de I 35 de pacienți cu ULMCAD tratat de PCl, dintre care 75 (55,6\%) au avut o strategie cu un singur stent (Grup A) și 60 (44,4\%) au avut o strategie cu două stenturi (Grupul B). Rezultate - Mai puțini pacienți din grupa A au avut un flux TIMI III la sfârșitul procedurii $(89,4 \%$ față de $100 \%, p=0,03)$ și revascularizare completă $(65,3 \%$ față de $88,3 \%, p=0,002)$. Am găsit o mortalitate timpurie mai mare în grupul A, fără a atinge o semnificație statistică $(13,4 \%$ față de $3,3 \%, p=0,1)$. Rata mortalității la urmărirea de 4 ani a fost mai mare cu grupa $A$ după analiza multivariabilă (HR 0,36 ajustat, $\mathrm{Cl} 0,15-0,85, \mathrm{p}=0,02)$. Nu am găsit diferențe semnificative între grupuri în ceea ce privește evenimentul cardiac advers major (MACE) (HR 0,85 ajustat, $\mathrm{Cl} 0,34-\mathrm{I}, 48, \mathrm{p}=0,7)$ sau revascularizarea țintelor (TLR) $(H R$ I,37, $\mathrm{Cl} 0,42-4,47, \mathrm{p}=0,6)$ la 4 ani de urmărire. Concluzii Printre pacienții neselectați cu IPC ULMCAD, cu sau fară ACS, rata mortalității precoce este similară între strategia cu unul și două stenturi. Deși, ratele TLR de 4 ani și MACE au fost similare între cele două grupuri, rata de mortalitate cauzală de 4 ani a fost mai mică în grupul de strategie cu două stenturi.

Cuvinte cheie: intervenție coronariană percutanată, boală coronariană principală stângă, strategie cu un singur stent, strategie cu două stenturi.

\footnotetext{
' Department of Cardiology, ,Prof. C.C. Iliescu” Institute for Cardiovascular Diseases, Bucharest, Romania

${ }^{2}$ "Carol Davila" University of Medicine and Pharmacy, Bucharest, Romania

${ }^{3}$ Department of Cardiology, Tulcea County Hospital, Romania

${ }^{4}$ Colentina Clinical Hospital, Bucharest, Romania

${ }^{5}$ Faculty of Health Sciences, University of Bristol, United Kingdom
}

Contact address:

Lucian M. Predescu, MD

„Prof. Dr. C.C. Iliescu” Emergency Institute for Cardiovascular

Diseases 258 Fundeni Street, Bucharest, Romania.

E-mail: lucianpredescu@gmail.com 


\section{INTRODUCTION}

With the new developments in device technology, stent platforms and intracoronary imaging, percutaneous coronary intervention $(\mathrm{PCl})$ of unprotected left main coronary artery disease (ULMCAD) has become a safer and more efficient treatment method. Although, coronary artery bypass grafting (CABG) was the standard treatment for complex left main lesions, an increasing number of patients with ULMCAD are treated by $\mathrm{PCl}$. The increasing experience with complex left main $\mathrm{PCl}$ had led to more ULMCAD patients with severe comorbidities and high surgical risk to be treated by $\mathrm{PCl}^{2,3}$. Comparative studies of $\mathrm{PCl}$ and CABG for ULMCAD have showed similarities between the two revascularization methods concerning major adverse cardiac event (MACE) ${ }^{4,5}$. Most studies on left main bifurcation lesions found that treatment with a single stent strategy is superior to a two-stent strategy ${ }^{6-12}$. The need for planned two-stent strategy varies between studies from $30 \%$ to $70 \%{ }^{6,13}$. Some studies have shown that some two-stent techniques, like Double Kissing Crush technique, was associated with better outcomes than one-stent technique ". All those reports though have excluded patients with acute coronary syndrome (ACS). As such there is a significant gap in knowledge when it comes to the treatment of patients presenting in an acute setting with a ULMCAD.

\section{AIM}

The aim of current study was to compare the 4-year outcomes of unselected patients with ULMCAD treated by $\mathrm{PCl}$ with a one-stent or two-stent strategy including those presenting with ACS.

\section{MATERIAL AND METHODS}

\section{Study Population}

All patients with ULMCAD treated by $\mathrm{PCl}$ between January 2014 and December 2018 were selected from the electronic hospital records at the „Prof. Dr. C.C. Iliescu" Institute for Cardiovascular Diseases, Bucharest, Romania. Patients with a history of CABG and occluded grafts and patients presenting with ACS were included, also. Two young patients were excluded due to uncommon etiologies of left main (LM) stenosis: a case of coronary lesion secondary to tertiary syphilis and a case of spontaneous coronary dissection involving the LM. Only patients with complete data were included in the study. We included only patients with distal left main stenosis. Patients with ostial and mid-shaft left main stenosis were excluded. This resulted in a total of I 35 patients for which demographic, clinical, angiographic, procedural, post-procedural and outcome data were extracted from the hospital electronic records. Life status was verified using the National Insurance Agency Platform in June 2019 to identify possible out of hospital deaths. Data analysis was performed with the approval of the institutional ethics committees of the hospital involved.

\section{Study outcomes}

MACEs were defined as the occurrence of death, myocardial infarction (MI) or target lesion revascularizations (TLRs). ACS was defined as either unstable angina, non-ST segment elevation MI (NSTEMI) or ST segment elevation MI (STEMI). TLR was defined as repeated $\mathrm{PCl}$ for restenosis of the entire segment involving the implanted stent and the 5-mm distal and proximal borders adjacent to the stent. Stent thrombosis was defined on the basis of Academic Research Consortium definitions according to timing of presentation as early ( $0-30$ days), late ( $3 \mathrm{I}-360$ days), or very late $(>360 \text { days })^{14}$. Angiographic success was defined as residual stenosis of $<30 \%$ by visual estimation in the presence of Thrombosis in Myocardial Infarction (TIMI) flow grade 3. Complete revascularization was defined as any attempt to revascularize all diseased segments ( $\geq 2.5 \mathrm{~mm}$ in diameter). The diagnosis of periprocedural $\mathrm{MI}$ was made if after $\mathrm{PCl}$ there was an increase in CK-MB or troponin levels that was 5 times the upper normal level.

\section{Statistical analysis}

Frequencies are given as numbers and percentages, continuous values as median (inter-quartile range or minimum-maximum values). Population characteristics were compared using the Mann-Whitney $U$ test, Kruskall Wallis test and Fisher's exact test. Patients were divided into two main groups based on the $\mathrm{PCl}$ technique used: one group used a one stent technique (Group A), while the other used a two/three stent technique (Group B).

Early outcomes (mortality, stent thrombosis, need for intra-aortic balloon pump (IABP), access site complications) are based on known status at 30 days and presented as percentage. Late outcomes are estimated using the Kaplan Meier method. Late outcomes of interest are mortality, TLR and MACE.

Predictors of early outcomes were identified using univariable linear regression adjusted by acute coronary syndrome at the time of procedure. Multivariable 
analysis was not possible due to only 12 early events. Predictors of late outcomes (death, TLR) were identified using a combination of backward and forward stepwise multivariable Cox regression, including all variables with a univariable regression $p$ value of less than 0.1 and less than $<10 \%$ missing values. The statistically significant variables left in the final model were considered independent predictors. The Group A vs Group B variable was always kept in the model, as a variable of interest. Statistical analyses were done with STATA/SE I2.0 (StataCorp LP, College Station, TX).

\section{RESULTS}

A total of 135 patients undergoing left main $\mathrm{PCl}$ were included, age ranging from 33 to 86 years (median of 63 years). Group A consisted of $n=75$ (55.6\%), Group $B$ of the remaining $n=60$ (44.4\%). Detailed demographic and baseline clinical characteristics by group are presented in Table I. We found few significant differences among the two groups, namely more hypertensive patients in Group B (93.3\% Group B vs $76 \%$ Group A, p=0.009).

\begin{tabular}{|c|c|c|c|c|}
\hline & Group A & Group B & Total & p value \\
\hline Age, y (median, IQR) & $62(55-69)$ & $65(55-7 I)$ & $63(55-7 I)$ & 0.2 \\
\hline Male & $55(73.3)$ & $43(7 I .7)$ & $98(72.6)$ & 0.8 \\
\hline \multicolumn{5}{|l|}{ Cardiovascular Risk factors } \\
\hline Hypertension & $57(76)$ & $56(93.3)$ & $113(83.7)$ & 0.009 \\
\hline Dyslipidemia & $64(86.5)$ & $5 I(85)$ & II5 (85.8) & 0.8 \\
\hline Diabetes & $17(23)$ & $19(31.7)$ & $36(26.9)$ & 0.3 \\
\hline Obesity & $15(20)$ & $20(33.3)$ & $35(25.9)$ & 0.1 \\
\hline \multicolumn{5}{|l|}{ Smoking status } \\
\hline Active smoker & $27(36)$ & $12(20)$ & $39(28.9)$ & 0.06 \\
\hline Former smoker & $10(13.3)$ & $15(25)$ & $25(18.5)$ & \\
\hline \multicolumn{5}{|l|}{ Patient history } \\
\hline ACS & $29(39.2)$ & $24(40)$ & $53(39.6)$ & 0.5 \\
\hline Unstable angina & $\mathrm{I}(3.4)$ & $4(16.7)$ & $5(9.4)$ & \\
\hline NSTEMI & $6(20.7)$ & $7(29.2)$ & $13(24.5)$ & \\
\hline STEMI & $22(75.9)$ & $13(54.1)$ & $35(66.1)$ & 0.2 \\
\hline $\mathrm{PCl}$ & $15(20)$ & $17(28.3)$ & $32(23.7)$ & 0.2 \\
\hline Atrial Fibrillation & $8(10.8)$ & $10(16.7)$ & $18(13.4)$ & 0.4 \\
\hline Stroke/TIA & $8(10.7)$ & $6(10)$ & $14(10.4)$ & 0.6 \\
\hline Bleeding & $\mathrm{I}(\mathrm{I} .4)$ & $4(6.7)$ & $5(3.7)$ & 0.2 \\
\hline COPD & $2(2.7)$ & $0(0)$ & $2(1.5)$ & 0.5 \\
\hline PAD & $13(17.6)$ & $8(13.3)$ & $21(15.7)$ & 0.6 \\
\hline Neoplasm & $5(6.8)$ & $3(5)$ & $8(6)$ & 0.7 \\
\hline \multicolumn{5}{|l|}{ Clinical presentation } \\
\hline Stable angina & $34(45.3)$ & $31(51.6)$ & $65(48.2)$ & \\
\hline Unstable angina & $13(17.3)$ & $14(23.3)$ & $27(20)$ & \\
\hline NSTEMI & $6(8)$ & $7(I I .7)$ & $13(9.6)$ & \\
\hline STEMI & $14(18.7)$ & $4(6.7)$ & $18(13.3)$ & \\
\hline ACS with cardiogenic shock & $8(10.6)$ & $4(6.7)$ & $12(8.9)$ & 0.2 \\
\hline Atrial Fibrillation & $6(8.2)$ & $6(10)$ & $12(8.9)$ & 0.8 \\
\hline LVEF, \% (median, IQR) & $45(35-55)$ & $50(35-60)$ & $45(35-55)$ & 0.1 \\
\hline \multicolumn{5}{|l|}{ LV systolic disfunction } \\
\hline None $(>=50 \%)$ & $35(46.7)$ & $31(53.4)$ & $66(49.6)$ & \\
\hline Mild (40-49\%) & $16(21.3)$ & $12(20.7)$ & $28(2 I . I)$ & \\
\hline Moderate (30-39\%) & $13(17.3)$ & II (19) & $24(18)$ & \\
\hline Severe $(<30 \%)$ & II (I4.7) & $4(6.9)$ & $15(11.3)$ & 0.6 \\
\hline \multicolumn{5}{|l|}{ Blood samples pre-PCI } \\
\hline $\mathrm{HB}, \mathrm{g} / \mathrm{dl}$ (median, IQR) & $13.7(12.7-15)$ & $14.4(12.3-14.7)$ & $13.7(12.3-14.8)$ & $13.7(12.7-15)$ \\
\hline Troponin, ng/ml (median, IQR) & $0.2(0.03-1.3)$ & $0.6(0.03-3.1)$ & $0.2(0.03-2.2)$ & $0.2(0.03-1.3)$ \\
\hline Creatinine clearance, $\mathrm{ml} / \mathrm{min} / \mathrm{I} .73 \mathrm{~m}^{2}$ (median, IQR) & $86(64-97)$ & 77 (64-93) & $81(64-96)$ & $86(64-97)$ \\
\hline
\end{tabular}


SYNTAX, SYNTAX II (PCl and CABG, with respective predicted mortality) and EuroSCORE II scores did not differ between the two groups. Mean SYNTAX and SYNTAX II score were 21 and 31.9, respectively, in Group A and 23.5 and 32.1, respectively, in Group B.

\section{Angiographic characteristics}

Table 2 shows the main angiographic findings by used technique. As expected, true bifurcation lesions (Medina $1 / I / I$ and $0 / I / I$ ) were more frequent in Group $B$, as are those involving the $L C X(I / 0 / I$ and $0 / 0 / I)$. Medina $0 / 1 / 0$ or those involving the LAD $(1 / 0 / 0,1 / 1 / 0)$ were more frequent in Group A.

\begin{tabular}{|c|c|c|c|c|}
\hline & Group A & Group B & Total & p value \\
\hline \multicolumn{5}{|l|}{ Arterial access site } \\
\hline Radial & $14(18.7)$ & $17(28.3)$ & $31(23)$ & 0.2 \\
\hline Femoral & $61(81.3)$ & $43(71.7)$ & $104(77)$ & 0.2 \\
\hline \multicolumn{5}{|l|}{ Lefit main lesion localization } \\
\hline Distal & $65(86.7)$ & $47(78.4)$ & $112(83)$ & \\
\hline Ostial and distal & $2(2.7)$ & $2(3.3)$ & $4(3)$ & \\
\hline Whole length & $8(10.7)$ & $11(18.3)$ & $19(14)$ & 0.4 \\
\hline Bifurcation & $56(74.7)$ & 49 (8I.7) & $105(77.8)$ & 0.3 \\
\hline Trifurcation & $19(25.4)$ & II (18.3) & $30(22.2)$ & 0.4 \\
\hline \multicolumn{5}{|l|}{ Other lesions } \\
\hline None & $28(37.3)$ & $18(30)$ & $46(34.1)$ & \\
\hline One vessel & $20(26.7)$ & $20(33.3)$ & $40(29.6)$ & \\
\hline Two vessels & $19(25.3)$ & $18(30)$ & $37(27.4)$ & \\
\hline Three vessels & $8(10.7)$ & $4(6.7)$ & $12(8.9)$ & 0.6 \\
\hline Chronic total obstruction & $20(26.6)$ & II (18.3) & $31(22.9)$ & 0.3 \\
\hline LAD ostium affected & $51(68)$ & $44(73.3)$ & $95(70.4)$ & 0.6 \\
\hline CX ostium affected & $15(20)$ & $45(75)$ & $60(44.4)$ & $<0.001$ \\
\hline LAD non-ostial & $35(46.7)$ & $26(43.3)$ & $61(45.2)$ & 0.7 \\
\hline CX non-ostial & $15(20)$ & $20(33.3)$ & $35(25.9)$ & 0.1 \\
\hline RCA lesion & $24(32)$ & $19(31.7)$ & $43(31.8)$ & 0.6 \\
\hline \multicolumn{5}{|c|}{ Left main lesion characteristics } \\
\hline Diffuse lesion & $35(46.7)$ & $37(61.7)$ & $72(53.3)$ & 0.1 \\
\hline Eccentric lesion & $57(76)$ & $44(73.3)$ & $101(74.8)$ & 0.8 \\
\hline Calcified lesion & $18(24)$ & $23(38.3)$ & $41(30.4)$ & 0.09 \\
\hline Ulcerated lesion & $25(33.3)$ & II (I8.3) & $36(26.7)$ & 0.05 \\
\hline Carina implicated & $7(9.3)$ & $2(3.3)$ & $9(6.7)$ & 0.3 \\
\hline \multicolumn{5}{|l|}{ Medina classification } \\
\hline $\mathrm{I} / \mathrm{I} / \mathrm{I}$ & $9(12)$ & $25(42.4)$ & $34(25.2)$ & \\
\hline $1 / 0 / 0$ & $19(25.3)$ & $4(6.7)$ & $23(17)$ & \\
\hline $1 / 1 / 0$ & $12(16)$ & $4(6.7)$ & $16(11.8)$ & \\
\hline $1 / 0 / 1$ & $2(2.7)$ & $7(11.7)$ & $9(6.7)$ & \\
\hline $0 / 1 / 0$ & $30(40)$ & $7(11.7)$ & $37(27.4)$ & \\
\hline $0 / 1 / 1$ & $2(2.7)$ & $8(13.3)$ & $10(7.4)$ & \\
\hline $0 / 0 / 1$ & $\mathrm{I}(\mathrm{I} .3)$ & $5(8.3)$ & $6(4.4)$ & $<0.001$ \\
\hline True bifurcation $(\mathrm{I} / \mathrm{I} / \mathrm{I}, 0 / \mathrm{I} / \mathrm{I})^{*}$ & II (I4.7) & $33(55.7)$ & $44(32.6)$ & $<0.001$ \\
\hline LM take-off angle $<70$ degrees & $26(41.9)$ & $14(25.9)$ & $40(34.5)$ & 0.08 \\
\hline \multicolumn{5}{|l|}{ Bifurcation angle } \\
\hline$>90$ degrees & $9(15.5)$ & $3(5.6)$ & $12(10.7)$ & \\
\hline $70-90$ & $25(43.1)$ & $20(37)$ & $45(40.2)$ & \\
\hline $45-69$ & $7(12.1)$ & $12(22.2)$ & $19(17)$ & \\
\hline$<45$ & $17(29.3)$ & $19(35.2)$ & $36(32.1)$ & 0.2 \\
\hline LM stenosis, \% (median, IQR) & $55(12-80)$ & $50(22-76)$ & $50(20-77)$ & 0.9 \\
\hline LAD stenosis, \% (median, IQR) & $86(67-95)$ & $83(58-92)$ & $85(66-93)$ & 0.2 \\
\hline CX stenosis, \% (median, IQR) & $25(15-82)$ & $75(50-88)$ & $70(32-86)$ & 0.03 \\
\hline
\end{tabular}




\section{Procedural characteristics}

In 59 cases (40.4\%) the $\mathrm{PCl}$ procedure was performed during the same session as the diagnostic coronary angiogram. In the remaining cases the median time between the two procedures was II days. Six patients (4.1\%) were on mechanical support system with IABP prior to the $\mathrm{PCl}$ procedure. Femoral access was the preferred approach, in $88.9 \%$ of cases. Either a $6 \mathrm{~F}$
One vs two-stent technique for left main PCI

(50.4\%) or a 7F (48.2\%) guiding catheter was used in most procedures.

More details on the procedural steps, use of proximal optimization technique (POT) and kissing balloon post dilatation (KBPD) are shown in Table 3. Pre-dilatation of main vessel was done at nominal pressure in more cases among Group B patients. POT was used in similar proportions in both groups $(60 \%$ in Group $A$

\begin{tabular}{|c|c|c|c|c|}
\hline & Group A & Group B & Total & p value \\
\hline \multicolumn{5}{|l|}{ Arterial access site } \\
\hline Femoral & $67(89.3)$ & $53(88.3)$ & $120(88.9)$ & 0.7 \\
\hline Radial & $8(10.7)$ & $7(11.7)$ & $15(11.1)$ & 0.5 \\
\hline Pre-PCI IABP & $3(4)$ & $3(5)$ & $6(4.4)$ & 0.5 \\
\hline \multicolumn{5}{|l|}{ Guide catheter } \\
\hline $6 \mathrm{~F}$ & $45(60)$ & $23(38.3)$ & $68(50.4)$ & \\
\hline $7 F$ & $28(37.3)$ & 37 (6I.7) & $65(48.2)$ & \\
\hline $8 \mathrm{~F}$ & $2(2.7)$ & $0(0)$ & $2(1.5)$ & 0.008 \\
\hline Rotablation & $2(2.7)$ & $\mathrm{I}(\mathrm{I} .7)$ & $3(2.2)$ & 0.6 \\
\hline MV predilatation & $59(79.7)$ & $47(78.3)$ & $106(79.1)$ & 0.5 \\
\hline SB predilatation & $8(\mathrm{II})$ & $40(66.7)$ & $48(36.1)$ & $<0.001$ \\
\hline Predilatation at nominal & $19(32.2)$ & $21(44.7)$ & $40(37.7)$ & 0.2 \\
\hline Dissection after predilatation & $11(17.2)$ & $\mathrm{I} 5(3 \mathrm{I} .9)$ & $26(23.4)$ & 0.6 \\
\hline POT & $45(60)$ & $42(70)$ & $87(64.4)$ & 0.3 \\
\hline POT after stent & $35(46.7)$ & $33(55)$ & $68(50.4)$ & 0.4 \\
\hline POT after KBPD & II (I4.7) & $27(45)$ & $38(28.1)$ & $<0.001$ \\
\hline POT balloon diameter, mm (median, IQR) & $4(4-4.5)$ & $4(4-4.5)$ & $4(4-4.5)$ & \\
\hline KBPD & $14(18.7)$ & $48(80)$ & $62(45.9)$ & $<0.001$ \\
\hline TKBPD & $\mathrm{I}(\mathrm{I} .3)$ & $4(6.7)$ & $5(3.7)$ & 0.1 \\
\hline Stent underexpansion of $>30 \%$ & $8(10.7)$ & $6(10)$ & $14(10.4)$ & 0.6 \\
\hline IFR used pre-PCI & $4(5.3)$ & $2(3.3)$ & $6(4.4)$ & 0.7 \\
\hline IFR used post-PCl & $2(2.7)$ & $2(3.3)$ & $4(3)$ & 0.6 \\
\hline IVUS used pre-PCI & $2(2.7)$ & $0(0)$ & $2(1.5)$ & 0.5 \\
\hline IVUS used post-PCl & $10(13.3)$ & $16(26.7)$ & $26(19.3)$ & 0.08 \\
\hline \multicolumn{5}{|l|}{ SB residual stenosis } \\
\hline None & $36(49.3)$ & $47(78.3)$ & $83(62.4)$ & \\
\hline$<50 \%$ & $26(35.6)$ & $12(20)$ & $38(28.6)$ & \\
\hline$>50 \%$ & II (I5.I) & $\mathrm{I}(\mathrm{I} .7)$ & $12(9.1)$ & 0.001 \\
\hline Procedural success & $68(90.7)$ & $59(98.3)$ & $127(94.1)$ & 0.08 \\
\hline Complete revascularisation & $49(65.3)$ & $53(88.3)$ & $102(75.6)$ & 0.002 \\
\hline \multicolumn{5}{|l|}{ TIMI } \\
\hline $\mathrm{I}$ & $4(5.3)$ & $0(0)$ & $4(3)$ & \\
\hline 2 & $4(5.3)$ & $0(0)$ & $4(3)$ & \\
\hline 3 & $67(89.4)$ & $60(100)$ & $127(94)$ & 0.03 \\
\hline \multicolumn{5}{|l|}{ Peri-procedural complications } \\
\hline Hematoma & $4(5.3)$ & $2(3.3)$ & $6(4.4)$ & 0.7 \\
\hline Stroke/TIA & $0(0)$ & $\mathrm{I}(\mathrm{I} .7)$ & $\mathrm{I}(0.7)$ & 0.4 \\
\hline Myocardial infarction & $\mathrm{I}(\mathrm{I} .3)$ & $3(5)$ & $4(3)$ & 0.3 \\
\hline Atrial fibrillation & $\mathrm{I}(\mathrm{I} .3)$ & $\mathrm{I}(\mathrm{I} .7)$ & $2(1.5)$ & 0.4 \\
\hline Need for external electric shock & $2(2.7)$ & $2(3.3)$ & $4(3)$ & 0.6 \\
\hline Bradi-arrhytmia & $7(9.3)$ & $3(5)$ & $10(7.4)$ & 0.5 \\
\hline Death during $\mathrm{PCl}$ & $3(4)$ & $0(0)$ & $3(2.2)$ & 0.2 \\
\hline $\mathrm{CIN}^{*}$ & $6(13)$ & $5(12.8)$ & II (I2.9) & 0.6 \\
\hline
\end{tabular}


vs $70 \%$ in Group $B, p=0.3)$, but was done after KBPD more frequently in Group B (14.7\% in Group A vs $45 \%$ in Group B, $p<0.001$ ), owning in part to the higher use of KBPD in this group (18.7\% in Group A vs $80 \%$ in Group B, $p<0.001$ ).

Procedural success with TIMI 3 flow was achieved in $94.1 \%$ cases, with complete revascularization in $75.6 \%$ patients. Instantaneous wave-free ratio (iFR) was performed before the $\mathrm{PCl}$ in 6 cases (4.4\%) and after in 4 cases (3\%). In 2 cases (I.5\%) intravascular ultrasound (IVUS) was used before the procedure, while in 26 (I9.3\%) it was used after the procedure.

Technical outcomes and post-procedural complications by strategy group are summarized in Table 3 . Single stent technique (Group A) resulted in more side branch residual stenosis, fewer cases of complete revascularization and fewer cases of TIMI 3 flow at the end of the procedure. There were fewer successful procedures in Group A, but only approaching statistical significance $(90.7 \%$ vs $98.3 \%, p=0.08)$. There were no differences between the two groups in term of peri-procedural complications.

\section{Early outcomes}

There was an $8.8 \%$ early mortality ( 30 days mortality) $(n=12)$, with a $2.2 \%$ peri-procedural mortality $(n=3)$. We found a higher early mortality in Group A compared to Group $B$, without reaching statistical significance $(13.4 \%$ vs $3.3 \%, p=0.1)$.

Most deaths occurred in patients presenting with ACS $(n=I I)$ and mostly in patients complicated with cardiogenic shock $(n=8)$. As such, early mortality in non-ACS patients was $1.48 \%$ with no peri-procedural deaths.

Predictors of early mortality are summarized in Table 4. All values are from bivariable regression adjusted by presence of ACS since most deaths occurred in these patients. ACS itself is a strong predictor of early mortality. We found no significant differences in early mortality between Groups A and B. Patients with ACS had a II.93 times higher risk of early mortality when compared to non-ACS patients $(p=0.02), 59.49$ times higher if also in cardiogenic shock $(p<0.00 \mathrm{I})$.

\begin{tabular}{|c|c|c|c|c|c|c|}
\hline \multirow[t]{2}{*}{30 days mortality } & \multicolumn{3}{|c|}{ Univariable analysis } & \multicolumn{3}{|c|}{ ACS adjusted } \\
\hline & OR & Cl & $p$ value & OR & Cl & p value \\
\hline Group B vs Group A & 0.22 & $0.05 ; 1.06$ & 0.06 & 0.23 & $0.05 ; 1.13$ & 0.07 \\
\hline ACS at presentation & 11.93 & $1.49 ; 95.26$ & 0.02 & & & $\mathrm{~N} / \mathrm{A}$ \\
\hline History of atrial fibrillation & 4.44 & $1.15 ; 17.13$ & 0.03 & 4.66 & $1.11 ; 19.53$ & 0.02 \\
\hline ACS with cardiogenic shock & 59.49 & $|2.5 ; 283.1|$ & $<0.001$ & 36.66 & $6.89 ; 194 ; 89$ & $<0.001$ \\
\hline IAPB pre-PCI & 13.33 & $2.34 ; 75.79$ & 0.003 & 7 & $1.2 ; 40.82$ & 0.03 \\
\hline Atrial fibrillation at presentation & 7.06 & $174 ; 28.58$ & 0.006 & 6.29 & $1.4 ; 28.19$ & 0.02 \\
\hline LVEF at $\mathrm{PCl}$ & $0.89 / \%$ & $0.84 ; 0.94$ & $<0.001$ & 0.9 & $0.85 ; 0.96$ & 0.001 \\
\hline LVEF $<50 \%$ & 5.61 & I.18;26.7 & 0.03 & & & 0.07 \\
\hline LVEF $<30 \%$ & 19.77 & $5.1 ; 76.52$ & $<0.001$ & 12 & $2.93 ; 49.09$ & 0.001 \\
\hline Severe mitral regurgitation & 6.1 & $1.32 ; 28.22$ & 0.02 & 5.09 & $1.01 ; 25.55$ & 0.05 \\
\hline $\mathrm{Hb}$ pre- $\mathrm{PCl}$ value & $0.63 / \mathrm{g} / \mathrm{dl}$ & $0.43 ; 0.94$ & 0.02 & 0.64 & $0.44 ; 0.95$ & 0.03 \\
\hline CK-MB pre- $\mathrm{PCl}$ value & $1.28 / 100 \mathrm{U}$ & $1.02 ; 1566$ & 0.04 & & & 0.1 \\
\hline Creatinine clearance pre-PCl & $0.97 / \mathrm{ml} / \mathrm{min} / 1.73 \mathrm{~m}^{2}$ & $0.94 ; 0.99$ & 0.015 & & & 0.06 \\
\hline $\mathrm{CIN}^{*}$ & 8.87 & $1.52 ; 51.55$ & 0.02 & 10.63 & $1.68 ; 67.26$ & 0.01 \\
\hline LM stenosis degree & $1.02 / \%$ & $1 ; 1.04$ & 0.03 & & & 0.06 \\
\hline Predilatation at nominal & 5.46 & $1.05 ; 28.36$ & 0.04 & 6.9 & $1.26 ; 37.58$ & 0.03 \\
\hline POT & 0.24 & $0.06 ; 0.84$ & 0.03 & 0.26 & $0.07 ; 0.96$ & 0.04 \\
\hline KBPD & 0.22 & $0.46 ; 1.08$ & 0.06 & & & 0.08 \\
\hline Post-PCI LM diameter & $0.22 / \mathrm{mm}$ & $0.05 ; 1.05$ & 0.06 & 0.2 & $0.04 ; 0.98$ & 0.05 \\
\hline Post-PCI LAD diameter & $0.007 / \mathrm{mm}$ & $0.0006 ; 0.09$ & $<0.001$ & 0.01 & $0.0009 ; 0.13$ & $<0.001$ \\
\hline Post-PCI CX diameter & 0.37 & $0.19 ; 0.69$ & 0.002 & 0.34 & $0.17 ; 0.69$ & 0.003 \\
\hline Stent underexpansion of $>30 \%$ & 3.39 & $0.79 ;|4.4|$ & 0.1 & & & 0.2 \\
\hline SB residual stenosis $<50 \%$ & 2.03 & $0.97 ; 4.25$ & 0.06 & & & 0.2 \\
\hline Complete revascularization & 0.08 & $0.02 ; 0.32$ & 0.001 & 0.08 & $0.02 ; 0.32$ & $<0.001$ \\
\hline TIMI flow & $0.04 /$ level & $0.006 ; 0.22$ & $<0.001$ & 0.06 & $0.01 ; 0.33$ & 0.001 \\
\hline
\end{tabular}




\section{Late outcomes}

Mortality, TLR and MACE at 4 years were $21.9 \%, 14 \%$ and $32.5 \%$ overall, $9.6 \%, 15 \%$ and $24.1 \%$ in non-ACS patients and $33.3 \%, 12.8 \%$ and $40.1 \%$ respectively in ACS-patients, respectively. Unadjusted comparisons of mortality, TLR and MACE at 4 years between Group A and Group B are shown in Figure I. Mortality rate at 4-year follow up was higher in Group A compared to Group B after multivariable analysis (HR 0.36, $\mathrm{Cl}$ 0.15-0.85, $\mathrm{p}=0.02$ ).

Predictors of late mortality and coronary reintervention are summarized in Table 5 . In multivariable analysis, two/three stent technique (Group B) is associated with a threefold reduction in 4-year mortality compared to one stent technique (Group A). Current risk and complexity scores were not independent predictors of mortality at 4 years in this group, but cardiogenic shock, severe left ventricular disfunction hemoglobin level below $12 \mathrm{~g} / \mathrm{dl}$ and diffusely infiltrated left main were.

Diabetes, a non-ostial circumflex artery lesion at diagnostic and the use of a Stentys stent were independent predictors of TLR at 4 years. We found no significant differences between Group $A$ and Group $B$ in terms of MACE at 4 years when adjusting for predictors of death and TLR (adjusted HR 0.85, adjusted $P$ value 0.7$)$.

\section{DISCUSSIONS}

Our study included an unselected population with ULMCAD PCI without considering the clinical presentation. Patients presenting with ACS that had ULMCAD were not excluded. The aim of this study was to show the result of real-life practice of ULMCAD $\mathrm{PCl}$ in a high-volume working center with experience in treating elective and urgent patients with com-
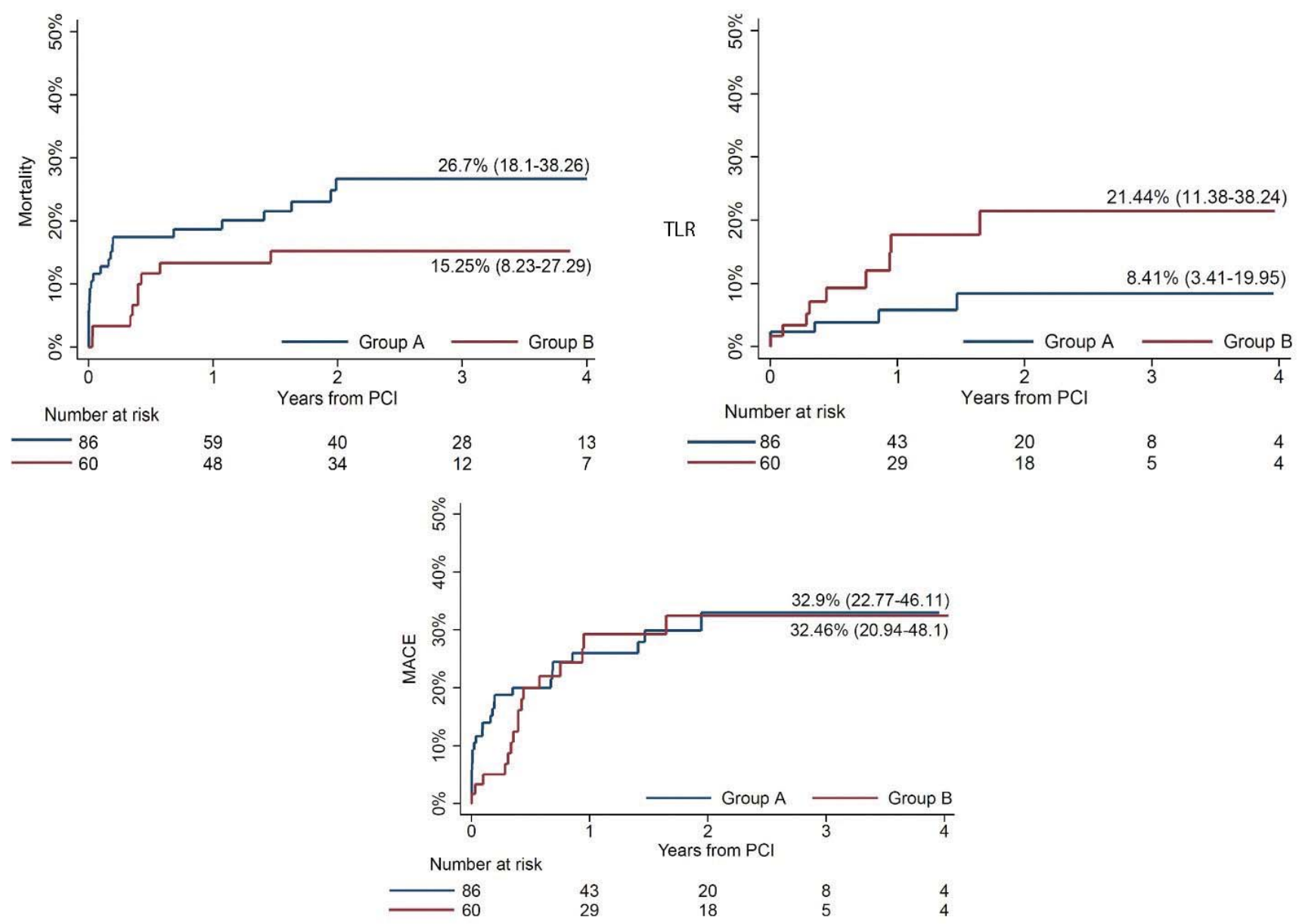

Figure I. Unadjusted comparisons of mortality, target lesion revascularization (TLR) and major adverse cardiac events (MACE) at 4 years according to the stent strategy in patients with unprotected left main coronary artery disease treated by percutaneous coronary intervention (Group $A$ - one stent strategy; Group B - two stent strategy). Curves represent Kaplan-Meier failure function. 


\begin{tabular}{|c|c|c|c|c|c|c|}
\hline \multirow[t]{2}{*}{ Long term mortality } & \multicolumn{3}{|c|}{ Univariable analysis } & \multicolumn{3}{|c|}{ Multivariable analysis } \\
\hline & HR & $\mathrm{Cl}$ & p value & HR & $\mathrm{Cl}$ & $P$ value \\
\hline Group B vs Group A & 0.5 & $0.23 ; 1.09$ & 0.08 & 0.36 & $0.15 ; 0.85$ & 0.02 \\
\hline Age & $1.03 / y$ & $0.99-1.06$ & 0.14 & & & \\
\hline Male gender & 1.89 & $0.72 ; 4.96$ & 0.19 & & & \\
\hline Hypertension & 0.48 & $0.21 ; 1.09$ & 0.08 & & & \\
\hline Former smoker & 0.3 & $0.07 ; 1.32$ & 0.11 & & & \\
\hline History of stroke & 2.33 & $0.95 ; 5.74$ & 0.06 & & & \\
\hline SYNTAX & I.05/unit & $1.02-1.1$ & 0.002 & & & \\
\hline SYNTAX-2 PCl & I.07/unit & I.04-I.I & $<0.001$ & & & \\
\hline SYNTAX-2 CABG & I.04/unit & $1.02-1.08$ & 0.003 & & & \\
\hline EUROSCORE II & I.16/unit & $1.11-1.22$ & $<0.001$ & & & \\
\hline ACS & 4.37 & $1.77 ; 10.75$ & 0.001 & & & \\
\hline ACS with cardiogenic shock & 13.74 & $6.15 ; 30.68$ & $<0.001$ & 6.09 & $2.08 ; 17.75$ & 0.001 \\
\hline Atrial Fibrillation at presentation & 2.67 & $1.01 ; 7.04$ & 0.05 & & & \\
\hline LVEF (\%) & $0.93 / \%$ & $0.9-0.96$ & $<0.001$ & & & \\
\hline LVEF $<30 \%$ & 12.52 & $5.93 ; 26.43$ & $<0.001$ & 5.77 & $2.26 ; 14.75$ & $<0.001$ \\
\hline Severe mitral regurgitation & 3.42 & $1.3 ; 9.02$ & 0.01 & & & \\
\hline Pulmonary hypertension* & 1.83 & $0.68 ; 4.94$ & 0.23 & & & \\
\hline $\mathrm{Hb}$ at $\mathrm{PCl}$ & $0.64 / \mathrm{g} / \mathrm{dl}$ & $0.52-0.80$ & $<0.001$ & & & \\
\hline $\mathrm{Hb}<12 \mathrm{~g} / \mathrm{dl}$ & 5.13 & $2.45 ; 10.72$ & $<0.001$ & 3.52 & $1.61 ; 7.67$ & 0.002 \\
\hline CK-MB at PCI* & 1.001 & $1-1.003$ & 0.06 & & & \\
\hline Creatinine clearance at $\mathrm{PCl}$ & 0.97 & $0.96-0.98$ & $<0.001$ & & & \\
\hline $\mathrm{CIN}^{*}$ & 5.33 & $1.95 ; 14.52$ & 0.001 & & & \\
\hline Associated two/three vessel disease & 1.62 & $1.02 ; 2.58$ & 0.04 & & & \\
\hline Diffuse LM plaques & 2.19 & $0.99 ; 4.82$ & 0.05 & 2.57 & $\mathrm{I} . \mathrm{I} ; 6.0 \mathrm{I}$ & 0.03 \\
\hline Ulcerated LM lesion & 3.07 & $1.48 ; 6.38$ & 0.003 & & & \\
\hline LM stenosis (\%) & $1.01 / \%$ & $1.005-1.03$ & 0.004 & & & \\
\hline Post PCl CX diameter $(\mathrm{mm})$ & $0.53 / \mathrm{mm}$ & $0.37 ; 0.75$ & $<0.001$ & & & \\
\hline Pre-PCI IABP & 8.65 & $3.21 ; 23.31$ & $<0.001$ & & & \\
\hline Post-PCI IABP & 4.15 & $1.57 ; 10.94$ & 0.004 & & & \\
\hline Predilation at nominal diameter & 2.98 & $1.3 ; 6.8 \mathrm{I}$ & 0.01 & & & \\
\hline POT used & 0.48 & $0.23 ; 1.007$ & 0.05 & & & \\
\hline Residual SB stenosis $>50 \%$ & 1.8 & $1.15 ; 2.83$ & 0.01 & & & \\
\hline Procedural succes & 0.17 & $0.06-0.45$ & $<0.001$ & & & \\
\hline \multirow[t]{2}{*}{ Complete revascularisation } & 0.26 & $0.13-0.55$ & $<0.001$ & & & \\
\hline & \multicolumn{3}{|c|}{ Univariable analysis } & \multicolumn{3}{|c|}{ Multivariable analysis } \\
\hline TLR & $\mathrm{HR}$ & $\mathrm{Cl}$ & $\mathrm{P}$ value & $\mathrm{HR}$ & $\mathrm{Cl}$ & $\mathrm{P}$ value \\
\hline Group B vs Group A & 2.22 & $0.74 ; 6.63$ & 0.15 & 1.37 & $0.42 ; 4.47$ & 0.6 \\
\hline Diabetes & 4.85 & $1.67 ; 14.09$ & 0.004 & 3.88 & $1.24 ; 12.12$ & 0.02 \\
\hline History of Atrial Fibrillation & 3.23 & $1.01 ; 10.34$ & 0.05 & & & \\
\hline Associated two/three vessel disease & 3.49 & $0.94 ; 12.93$ & 0.06 & & & \\
\hline Non ostial LCX lesion & 5.23 & $1.75 ; 15.63$ & 0.003 & 5.52 & $1.78 ; 17.15$ & 0.003 \\
\hline Calcified LM lesion & 2.77 & $0.97 ; 7.93$ & 0.06 & & & \\
\hline LAD stenosis (\%) & $1.01 / \%$ & $0.99-1.04$ & 0.1 & & & \\
\hline Stentys stent used & 3.07 & $0.96 ; 9.8$ & 0.06 & 3.61 & I.II;1I.74 & 0.03 \\
\hline MV stent diameter $(\mathrm{mm})$ & 0.27 & $0.04 ; 1.98$ & 0.2 & & & \\
\hline Stent underexpansion of $>30 \%$ & 2.75 & $0.76 ; 9.89$ & 0.12 & & & \\
\hline *>10\% missing value, not used in mul & & & & & & \\
\hline
\end{tabular}


plex LM lesions. The study included I 35 patients with ULMCAD PCl of which $51.8 \%$ presented with ACS. The study population was divided in two groups to compare different stent strategy for the left main $\mathrm{PCl}$ (one-stent strategy versus two-stent strategy). There are few reports of such comparison that included the patient presented with ACS.

Although, our study has included the patients presented with ACS, the early mortality was similar to the EXCEL (Evaluation of XIENCE Versus Coronary Artery Bypass Surgery for Effectiveness of Left Main Revascularization) trial $(8.8 \%$ in our study vs $8.1 \%$ in EXCEL trial) ${ }^{12,15}$. The 4-year mortality rate was significantly higher than in the NOBLE (Nordic-Baltic-British left main revascularisation) trial at 5 year follow up (mortality rate in our study was $21.9 \%$ versus $12 \%$ in NOBLE trial) $)^{16}$. When we excluded the patient presented with ACS, the long-term mortality and TLR rates were comparable in the two studies (mortality and TLR rate in our study were $9.6 \%$ and $15 \%$, respectively versus $12 \%$ and $16 \%$ in NOBLE trial) ${ }^{16}$.

As we expected, the two-stent strategy was used in more complex patients, with diffuse left main plaques, calcified left main lesions and with Medina I/I/I or $0 / 1 / 1$ lesion. POT use was similar among the two groups, but KBPD was used more frequently in the two-stent strategy group (18.7\% in Group A vs $80 \%$ in Group B, $\mathrm{P}<0.00 \mathrm{I}$ ). In univariable analysis POT, but not KBPD, was a predictor for early mortality. Neither POT nor KBPD were a predictor for long-term outcomes in the multivariable analysis.

Although we found a higher early mortality in patients with ULMCAD treated with one-stent strategy compared to two-stent strategy, it didn't reach the statistical significance ( $13.4 \%$ vs $3.3 \%, p=0.1)$, possibly in the context of low number of events. This can be since the group treated with a one-stent strategy included a bigger number of patients with ACS complicated with cardiogenic shock, which was a strong predictor and confounder of 30 days mortality. The results are consistent with other studies ${ }^{6,10,11,17}$.

In contrast to other studies, the 4-year mortality rate was significantly lower in the two-stent strategy group compared to the one-stent strategy group. No significant differences between the two group in terms of MACE at 4 years was found when adjusting for predictors of death and TLR (adjusted HR 0.85, adjusted $P$ value 0.7$)$. This findings can partially be explained by the lower number of patients in the Group A with TIMI III flow at the end of the procedure $(89.4 \%$ vs
$100 \%, p=0.03)$, with complete revascularization $(65.3 \%$ vs $88.3 \%, p=0.002)$ and with no residual side branch stenosis $(49.3 \%$ vs $78.3 \%, p=0.001)$. Considering this data, we can emphasize that the one-stent strategy group was a higher risk group with patients in a more severe clinical status although the anatomical complexity of LM lesion was lower in the one-stent strategy group. This finding can show that in a high-volume center with a big experience in performing $\mathrm{LM} \mathrm{PCl}$ the long-term outcomes can be driven more by the clinical condition of the patient and other patient related features, than maybe technical aspects. Nevertheless, the influence of an acute setting in left main $\mathrm{PCl}$ needs to be further investigated, as it might also contribute, by unknown mechanisms, to different outcomes than expected from studies looking at stable angina.

One important study limitation is the nonrandomized study design. Although it might have offered more data on restenosis, routine angiographic reevaluation is no longer recommended and was not performed.

\section{CONCLUSIONS}

Among patients with ULMCAD PCl with or without ACS, the early mortality rate is similar between one and two-stent strategy. Although, 4-year TLR and MACE rates were similar between the two groups, the 4-year mortality rate was lower in the two-stent strategy group. The optimal strategy in UPMLCA PCI outside stable angina requires further research.

\section{Conflict of interest: none declared.}

\section{References}

I. Predescu L.M, Zarma L, Platon P, et al, Current treatment of left main coronary artery disease, Cor et Vasa 58 (2016), pp. e328-e339, DOl information: 10.1016/j.crvasa.2015.05.007.

2. Park SJ, Ahn JM, Kim YH, Park DW, Yun SC, Yoon SH, et al. Temporal trends in revascularization strategy and outcomes in left main coronary artery stenosis: data from the ASAN Medical Center-Left MAIN Revascularization registry. Circulation Cardiovascular interventions. 2015;8(3):e001846.

3. Lassen JF, Burzotta F, Banning AP, Lefevre T, Darremont O, HildickSmith D, et al. Percutaneous coronary intervention for the left main stem and other bifurcation lesions: 12th consensus document from the European Bifurcation Club. Eurolntervention : journal of EuroP$\mathrm{CR}$ in collaboration with the Working Group on Interventional Cardiology of the European Society of Cardiology. 201 8; I3(I3): I540-53.

4. Cavalcante R, Sotomi Y, Lee CW, Ahn JM, Farooq V, Tateishi H, et al. Outcomes After Percutaneous Coronary Intervention or Bypass Surgery in Patients With Unprotected Left Main Disease. Journal of the American College of Cardiology. 2016;68(10):999-1009.

5. Nerlekar N, Ha FJ, Verma KP, Bennett MR, Cameron JD, Meredith $\mathrm{IT}$, et al. Percutaneous Coronary Intervention Using Drug-Eluting Stents Versus Coronary Artery Bypass Grafting for Unprotected Left Main Coronary Artery Stenosis: A Meta-Analysis of Randomized Trials. Circulation Cardiovascular interventions. 20I6;9(I2).

6. Pavani M, Conrotto F, Cerrato E, D'Ascenzo F, Kawamoto H, Nunez-Gil IJ, et al. Long-Term Outcomes of Different Two-Stent 
Techniques With Second-Generation Drug-Eluting Stents for Unprotected Left Main Bifurcation Disease: Insights From the FAILS-2 Study. The Journal of invasive cardiology. 2018;30(8):276-8I.

7. Tiroch K, Mehilli J, Byrne RA, Schulz S, Massberg S, Laugwitz KL, et al. Impact of coronary anatomy and stenting technique on long-term outcome after drug-eluting stent implantation for unprotected left main coronary artery disease. JACC Cardiovascular interventions. 2014;7(I):29-36.

8. Kim YH, Park DW, Ahn JM, Yun SC, Song HG, Lee JY, et al. Everolimus-eluting stent implantation for unprotected left main coronary artery stenosis. The PRECOMBAT-2 (Premier of Randomized Comparison of Bypass Surgery versus Angioplasty Using Sirolimus-Eluting Stent in Patients with Left Main Coronary Artery Disease) study. JACC Cardiovascular interventions. 2012;5(7):708-17.

9. Colombo A, Bramucci E, Sacca S, Violini R, Lettieri C, Zanini R, et al. Randomized study of the crush technique versus provisional sidebranch stenting in true coronary bifurcations: the CACTUS (Coronary Bifurcations: Application of the Crushing Technique Using Sirolimus-Eluting Stents) Study. Circulation. 2009;1 I9(I):7I-8.

10. Gao Z, Xu B, Yang Y, Qiao S, Wu Y, Chen T, et al. Comparison between one-stent versus two-stent technique for treatment of left main bifurcation lesions: A large single-center data. Catheterization and cardiovascular interventions : official journal of the Society for Cardiac Angiography \& Interventions. 2015;85(7): I 132-8.

II. Chen SL, Zhang Y, Xu B, Ye F, Zhang J, Tian N, et al. Five-year clinical follow-up of unprotected left main bifurcation lesion stenting: one-stent versus two-stent techniques versus double-kissing crush technique. Eurolntervention : journal of EuroPCR in collaboration with the Working Group on Interventional Cardiology of the European Society of Cardiology. 2012;8(7):803-I4.
12. Kandzari DE, Gershlick AH, Serruys PW, Leon MB, Morice MC, Simonton CA, et al. Outcomes Among Patients Undergoing Distal Left Main Percutaneous Coronary Intervention. Circulation Cardiovascular interventions. 2018; II (I0):e007007.

13. Chen SL, Santoso T, Zhang JJ, Ye F, Xu YW, Fu Q, et al. A randomized clinical study comparing double kissing crush with provisional stenting for treatment of coronary bifurcation lesions: results from the DKCRUSH-II (Double Kissing Crush versus Provisional Stenting Technique for Treatment of Coronary Bifurcation Lesions) trial. Journal of the American College of Cardiology. 20I I;57(8):9|4-20.

14. Cutlip DE et al, Academic research consortium. Clinical end points in coronary stent trials: A case for standardized definitions. Circulation 2007; II 15:2344-235I.

15. Stone GW, Sabik JF, Serruys PW, Simonton CA, Genereux P, Puskas J, et al. Everolimus-Eluting Stents or Bypass Surgery for Left Main Coronary Artery Disease. The New England journal of medicine. 2016;375(23):2223-35.

16. Makikallio T, Holm NR, Lindsay M, Spence MS, Erglis A, Menown IB, et al. Percutaneous coronary angioplasty versus coronary artery bypass grafting in treatment of unprotected left main stenosis (NOBLE): a prospective, randomised, open-label, non-inferiority trial. Lancet. 2016;388(1006I):2743-52.

17. Palmerini T, Marzocchi A, Tamburino C, Sheiban I, Margheri M, Vecchi G, et al. Impact of bifurcation technique on 2-year clinical outcomes in 773 patients with distal unprotected left main coronary artery stenosis treated with drug-eluting stents. Circulation Cardiovascular interventions. 2008; I(3): 185-92. 